\title{
Re-do lung transplantation: keys to success
}

\author{
Lara Schaheen ${ }^{1}$, Jonathan D'Cunha ${ }^{2}$ \\ ${ }^{1}$ Norton Thoracic Institute, St Joseph's Hospital and Medical Center, Phoenix, AZ, USA; ${ }^{2}$ Department of Cardiothoracic Surgery, Mayo Clinic \\ Arizona, Phoenix, AZ, USA \\ Correspondence to: Jonathan D'Cunha, MD, PhD. Department of Cardiothoracic Surgery, Mayo Clinic Arizona, 5777 E Mayo Blvd, Phoenix, AZ \\ 85054, USA. Email: DCunha.Jonathan@mayo.edu. \\ Provenance: This is an invited article commissioned by the Academic Editor Xianglin Hu (Department of Pulmonary Medicine, Zhongshan Hospital, \\ Fudan University, Shanghai, China). \\ Comment on: Wallinder A, Danielsson C, Magnusson J, et al. Outcomes and Long-term Survival After Pulmonary Retransplantation: A Single-Center \\ Experience. Ann Thorac Surg 2019;108:1037-44.
}

Submitted Dec 10, 2019. Accepted for publication Dec 24, 2019.

doi: $10.21037 /$ jtd.2019.12.104

View this article at: http://dx.doi.org/10.21037/jtd.2019.12.104

Lung transplantation (LTx) is a life-saving treatment for patient's afflicted by end-stage pulmonary disease. The field has enjoyed tremendous growth over the past several decades with an improvement in outcomes and survival. Increasing experience and improvements in survival has led to an increased frequency of patients being considered for pulmonary re-transplantation. Although survival following lung re-transplantation (ReLTx) has had definite improvement in recent years, outcomes remain poorer than with primary LTx. Refinements in candidate selection for re-transplantation may lead to an improvement in outcomes following re-transplantation and better understanding of which patient's should be offered re-transplantation.

Many recipients of LTx are young when transplanted and often are referred for consideration of re-transplantation as their grafts fail from chronic rejection. With a median survival of 2.5 years after re-transplantation, there is much to be gained from trying to elucidate risk factors for a poor outcome in this population. This is clearly an important question as the International Society for Heart and Lung Transplantation data would suggest that the number of retransplants is on the rise and donor organs remain in limited supply. One also must always ask the ethical question in a re-transplant that does not do well if that set of lungs would have been better served into a first-time recipient.

As a part of this and this commentary, we feel the area of ReLTx is plagued by three pressing questions:

(I) Who should we re-transplant?

(II) How do we stage the risk of the progression of a listed patient?

(III) Are all re-transplants the same?

\section{Who should we re-transplant?}

This is a key question in the area of patient in need of a reoperative LTx. Each center that performs LTx typically has their own criteria for listing a patient needing a re-transplant. Often these include multifactorial decisions, but center around why the original transplant failed, the age of the patient, comorbidities, functional status, and psychosocial factors. The article recently published by Wallinder and colleagues in the Annals of Thoracic Surgery seeks to shed some light on the factors influencing the decision to re-transplant (1). In this work, the authors evaluated their experience with re-transplantation at their institution with the goal of identifying negative predictors of outcome for this high-risk procedure. Over a 27-year period the authors performed 49 ReLTx. Outcomes were compared to the primary LTx group from their institution which had 635 patients included. Heart-LTxs were excluded in the analysis. In their analysis, they found that median survival after re-transplantation was only 2.5 years and recipients were younger in age. Risk factors for poor outcome were the same as primary LTx and included recipient sex, age, diabetes, pre-operative mechanical ventilation, impaired renal function, and need for preoperative ECMO. Importantly, early ReLTx (within 1 year) as an indication for the procedure was met was reduced survival. The other interesting finding on deeper dissection of the work 
demonstrated that a double lung ReLTx had a hazard ratio of 3.5. The authors suggest that in the context of their work and others that single lungs should be placed into patients who need a ReLTx (a single lung in the contralateral side of a patient who previously had a single LTx). In their dataset of 49 LTxs, 39 patients were done as a SLTx including 21 procedures on the contralateral side after initial SLTx. Thus, only 10 patients in their ReLTx group had true bilateral procedures. Although a SLTx on the contralateral side of a patient who had a SLTx in the past, the technical aspects of the procedure are quite different than a double ReLTx and as we consider these findings, attention needs to be called to this nuance.

This concept of early mortality from an early ReLTx has been validated previously in the work from the Duke group that looked at the United Network for Organ Sharing registry. In their study of 456 ReLTx in the lung allocation score era that underwent propensity score matching, recipients undergoing early ReLTx, within 90 days or primary procedure, had a significant survival disadvantage (2). Other factors conferring worse outcomes included intensive care unit admission, single LTx, poor functional status, and primary graft dysfunction as the indication for ReLTx.

\section{How do we stage the risk of the progression of a listed patient?}

The second important question that needs addressing is this patient population that patients have progressive disease that is dynamic. In our practice, we have made it a point generally to say that a patient who needs to be re-transplanted needs to "walk into clinic" to be evaluated. A debilitated inpatient is not someone who generally will do well with a high-risk operation. This is not surprising. More commonly, and importantly, once a patient is listed, we need to understand if they remain candidates for transplantation throughout the time they are listed as their disease may progress. To gain greater insight into this question, we performed a propensityadjusted risk analysis on adult re-LTx patients and found that patients bridged with extracorporeal membrane oxygenation (ECMO) to transplant had a very poor 30 day survival rate when compared to those that were not bridged $(67.3 \% \mathrm{vs}$. $91.2 \%, \mathrm{P}=0.0002$, respectively) (3).

\section{Are all "re-transplants" created equal?}

We do want to call special attention to this area as it is well established that ReLTx portends a more complex postoperative course and lower peak lung function (4). In the work being reviewed here and put forth by Wallinder and colleagues, in their dataset of 49 LTxs, 39 patients were done as a SLTx including 21 procedures on the contralateral side after initial SLTx. We believe that future studies should carve out these types of procedures from being considered a "re-transplant." Although we can appreciate the fact that these are cases that come to the surgeon with their own challenges, the surgical risk incurred is well-accepted at the technical level to be quite lower. The risks incurred are those typically of patient protoplasm and the medical course they have had leading up to transplant. Major risk factors would include nutritional status, immunosuppressive medications, and overall performance status. From a surgical standpoint, the most challenging technical consideration is the intraoperative management of single lung ventilation on the side that is failing and it would not be unusual to require extracorporeal support during this time. This of course increases the risk profile overall.

The other concept that we need to touch on in this section is the donor: recipient relationship. This also has the potential to contribute to the concept of how not all transplants are created equal. Although there is not much data in this realm outside of what is known for primary lung transplant recipients, most experts accept the fact that as complexity of the transplant increases, the better the donor needs to be. Studies put forth like the one recently by Aurån and colleagues suggest that donors with an age of 55 and older in cystic fibrosis recipients had inferior survival and long ICU length of stay. In their study, other recipients did not have inferior survival (5). The potential translation here to ReLTx is that typically these recipients are those with cystic fibrosis who have progressive chronic rejection in need of a second pair of lungs.

All in all, the study published by Wallinder is timely and thought-provoking as we have learned much about ReLTx through these types of studies recently. Our take home points are as follows:

(I) Proceed with great reservation on a ReLTx recipient within one year of the primary procedure;

(II) Functional status is key and it is best to have a ReLTx recipient "walk into clinic";

(III) If a listed ReLTx deteriorates and requires ECMO, proceed with great caution. Patients going on veno-venous ECMO can likely be bridged, but the mortality from arterio-venous ECMO as a bridge is essentially prohibitive from maintaining candidacy in our eyes at this time;

(IV) Pick a good donor set of lungs when the time 
comes. Good donor equals good outcome.

\section{Acknowledgments}

None.

\section{Footnote}

Conflicts of Interest: The authors have no conflicts of interest to declare.

Ethical Statement: The authors are accountable for all aspects of the work in ensuring that questions related to the accuracy or integrity of any part of the work are appropriately investigated and resolved.

\section{References}

1. Wallinder A, Danielsson C, Magnusson J, et al.

Cite this article as: Schaheen L, D'Cunha J. Re-do lung transplantation: keys to success. J Thorac Dis 2019;11(12):56915693. doi: $10.21037 /$ jtd.2019.12.104
Outcomes and Long-term Survival After Pulmonary Retransplantation: A Single-Center Experience. Ann Thorac Surg 2019;108:1037-44.

2. Osho AA, Castleberry AW, Snyder LD, et al. Differential outcomes with early and late repeat transplantation in the era of the lung allocation score. Ann Thorac Surg 2014;98:1914-20; discussion 1920-1.

3. Hayanga JW, Aboagye JK, Hayanga HK, et al. Extracorporeal membrane oxygenation as a bridge to lung re-transplantation: is there a role? J Heart Lung Transplant 2016;35:901-5.

4. Halloran K, Aversa M, Tinckam K, et al. Comprehensive outcomes after lung retransplantation: a single-center review. Clin Transplant 2018;32:e13281.

5. Aurån H, Durheim MT, Dellgren G, et al. Effect of donor age on outcome of lung transplantation stratified by recipient diagnosis: a Nordic multicenter study. Transplantation 2019;103:807-14. 